\title{
Online Dispatch of Shared Energy Storage with Remote Renewable Generations
}

\author{
Shenghe Wang ${ }^{1}$, Jinzhong $\mathrm{Li}^{2}$, Yuguang $\mathrm{Xie}^{2}$, Jiayu $\mathrm{Bai}^{3}{ }^{*}$, Bo $\mathrm{Gao}^{2}$, and Shengwei $\mathrm{Mei}^{3}$ \\ ${ }^{1}$ State Grid Anhui Electric Power CO., LTD., Hefei 230022, China \\ ${ }^{2}$ State Grid Anhui Electric Power Research Institute, 230601 Hefei, China \\ ${ }^{3}$ State Key Lab of Control and Simulation of Power Systems and Generation Equipments (Dept. of Electrical Engineering), Tsinghua \\ University, 100084 Beijing, China
}

\begin{abstract}
The sustained penetration of wind and solar generation is conducive to alleviating the energy crisis and environmental pollution. However, the finite capacity of transmission corridor limits the delivery of remote renewables and leads to curtailment. Energy storage plays an important role in renewables accommodation and improving equipment utilization, and shared energy storage can magnify the benefits through a temporal and spatial complementary. This paper proposes an online dispatch approach of energy storage shared by multiple renewable plants. The uncertainty and non-anticipativity issue are addressed via a two-stage decision-making process. The day-ahead scheduling determines the allowable energy and power levels of shared energy storage; the real-time online dispatch decides the wait-and-see strategies of charging and discharging power based on a single-period optimization model constrained by the pre-scheduled bounds. The case study verifies the effectiveness of the proposed method.
\end{abstract}

\section{Instruction}

In response to the worldwide challenges of energy crisis and environmental pollution, renewable energy represented by wind and photovoltaic (PV) power is developing rapidly in recent years. In China, the installed capacity of renewable generation has surged to $4000 \mathrm{GW}$ by 2020 and is projected to be increased to 12 billion $\mathrm{kW}$ by 2030 . Although the penetration of renewable energy accelerates grid decarbonization and promotes society's sustainable development, the inherent volatility and stochasticity of renewables bring huge challenges to power system dispatch. Moreover, the unbalanced geographical distribution and the insufficient capacity of supporting transmission channels result in massive resource abandonment. Energy storage is a flexible resource to compensate for the mismatch of renewables and load demand and mitigate the curtailment [1].

Motivated by the success of sharing economy, shared energy storage is attracting increasing attention. Considering the high cost and low utilization ratio of energy storage in individual frameworks, shared energy storage exploits the temporal and spatial demand complementary of different users and brings about cost reduction [2]. The shared energy storage can be implemented on the demand-side or generation side. The day-ahead operation of shared energy storage with PV power producers is studied in [3], and the sensitivitybased allocation ratio is adopted for profit distribution. In [4], a combinatorial auction mechanism is induced to the allocation of residential shared energy storage, wherein the capacity, energy storage level, charging and discharging power are taken as multi-sources to be auctioned. [5] pays attention to the electricity losses during the sharing process and develops a hierarchical capacity planning approach for community-level shared batteries. Taking into account the price uncertainty, [6] proposes a sharing strategy of energy storage with two users based on a robust optimization model. Based on the equilibrium approach, [7] presents a pricing and dispatch strategy of a residential system with shared energy storage and multiple consumers.

Considering the randomness and limited predictability of renewables, numerous methods have been proposed for the optimal dispatch of energy storage with renewable energy. Stochastic optimization [8] and robust optimization [9] are classical approaches to address the uncertainty issues, and both methods are formulated based on offline data, such as forecast and historical data while ignoring the non-anticipativity effect. However, the uncertainty is observed period by period and may not be realized as anticipated, thus the anticipative schedules generated by conventional two-stage stochastic/robust optimization may be infeasible in practical operation [10]. To guarantee the causality of decision-making, the scheduling strategy is supposed to be independent of future information.

To resolve the uncertainty and non-anticipatively issues of shared energy storage dispatching with renewable generations, this paper proposes an online dispatch method, which is composed of two stages of decision-making. The day-ahead dispatch pre-schedules

\footnotetext{
$\overline{{ }^{*} \text { Corresponding author: Email: bai_jiayu01@163.com, Tel: +86 } 18200542600 .}$
} 
the allowable state-of-charge (SoC) ranges of shared energy storage; the real-time scheduling determines the wait-and-see decisions of charging and discharging power based on a single-period optimization model constrained by the pre-prespecified bounds without any future information.

The remainder of the paper is organized as follows. Section 2 presents the online dispatch framework of the shared energy storage with renewable plants and establishes the models of non-anticipative day-ahead scheduling and online dispatch. The case study is analyzed in Section 4 and conclusions are drawn in Section 5.

\section{Problem Formulation}

\subsection{Online dispatch framework}

The structure of an integrated system with renewables and shared energy storage is shown in Fig. 1. The renewables, including wind power and photovoltaic power, are sold to the grid through the transmission line. Considering the fluctuation of renewable outputs, curtailment takes place in abundant scenarios due to line capacity limitation, and the under-utilization of devices results from insufficient outputs. The energy storage is shared by the solar plants and wind plants to cope with uncertainty and smooth the output.

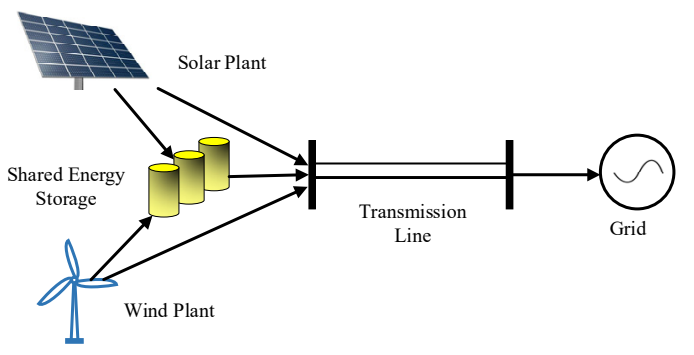

Fig.1 System structure.

In the day-ahead stage, the allowable SoC ranges and the permitted charging/discharging level of shared energy storage is determined based on a rough prediction of the renewable outputs; in the real-time stage, the scheduling moves on hour by hour, and the strategy is determined by a single-period optimization problem with the prespecified bounds after observing the real output of renewables. The online dispatch framework is displayed as follows.

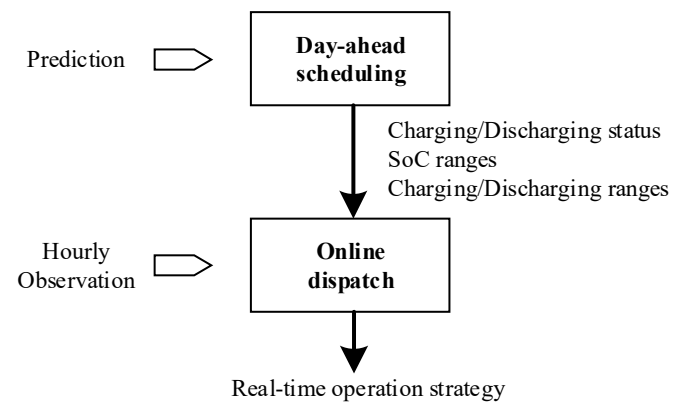

Fig.2 Online dispatch framework.

\subsection{Non-anticipative day-ahead scheduling}

The uncertainty of accessible renewable outputs is described via the following box set.

$$
U^{r a}=\left\{p_{i, t}^{r a} \mid \underline{p}_{i, t}^{r a} \leq p_{i, t}^{r a} \leq \bar{p}_{i, t}^{r a} ; \forall i, t\right\}
$$

where the subscript $i$ is the index of renewable plant, and subscript $t$ is the index of time slot. $p_{i, t}^{r a}$ is the uncertain output of each renewable plant, $\underline{p}_{i, t}^{r a}$ and $\bar{p}_{i, t}^{r a}$ are the predicted lower and upper bounds. The minimal possible total output $\underline{p}_{i, t}^{r \Sigma}$ can be calculated by $\sum_{i}^{I} \underline{p}_{i, t}^{r}$, and the maximal possible total output $\bar{p}_{i, t}^{r \Sigma}$ equals $\sum_{i}^{I} \bar{p}_{i, t}^{r}$, where $I$ is the number of renewable plants. Then, the uncertainty of total renewablw output $p_{t}^{r a \Sigma}$ can be judged by

$$
U^{r a \Sigma}=\left\{p_{t}^{r a \Sigma} \mid \underline{p}_{t}^{r a \Sigma} \leq p_{t}^{r a \Sigma} \leq \bar{p}_{t}^{r a \Sigma} ; \forall t\right\}
$$

Consider the curtailment of renewables, the uncertainty set of real accommodated renewables is adjusted by

$$
U^{r}=\left\{p_{t}^{r \Sigma} \mid \min \left\{\bar{p}_{t}^{r a \Sigma}-h_{t}, \underline{p}_{t}^{r a \Sigma}\right\} \leq p_{t}^{r \Sigma} \leq \bar{p}_{t}^{r a \Sigma}-h_{t} ; \forall t\right\}
$$

where $h_{t}$ is the curtailment level, and is determined in the day-ahead scheduling. Two vertex scenarios denoted by $S 1$ and $S 2$ are picked from the uncertainty realizations:

$$
p_{t, S 1}^{r \Sigma}=\bar{p}_{t}^{r a \Sigma}-h_{t} ; \forall t
$$

which represents the maximal renewable output ;

$$
p_{t, S 2}^{r \Sigma}=\min \left\{\bar{p}_{t}^{r a \Sigma}-h_{t}, \underline{p}_{t}^{r a \Sigma}\right\} ; \forall t
$$

which represents the minimal renewable output. The dayahead dispatch problem is formulated as follows.

$$
\begin{aligned}
& \max _{\substack{\mu_{t}^{c}, \mu_{t}^{d}, h_{t}, \tilde{p}_{t, s}^{r \Sigma} \\
E_{\min } E_{E^{\max }}}} \sum_{t=1}^{T} C_{t} \sum_{s=1}^{S} \omega_{s}\left(\tilde{p}_{t, s}^{r \Sigma}-p_{t, s}^{c}+p_{t, s}^{d}\right)
\end{aligned}
$$

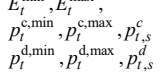

$$
\begin{aligned}
& \text { s.t. } \alpha^{L} p^{L} \leq \tilde{p}_{t, s}^{r \Sigma}-p_{t, s}^{c}+p_{t, s}^{d} \leq p^{L} ; \forall t, s \\
& \tilde{p}_{t, s}^{r \Sigma}=\min \left\{\bar{p}_{t}^{r a \Sigma}-h_{t}, p_{t, s}^{r \Sigma}\right\} ; \forall t, s \\
& 0 \leq h_{t} \leq \bar{p}_{t}^{r a \Sigma} \\
& p_{t}^{c, \text { min }} \leq p_{t, s}^{c} \leq p_{t}^{c, \text { max }} ; \forall t, s \\
& p_{t}^{d, \text { min }} \leq p_{t, s}^{d} \leq p_{t}^{d, \text { max }} ; \forall t, s \\
& E_{t}^{\min }=E_{t-1}^{\min }-p_{t}^{d, \text { max }} / \eta^{d}+\eta^{c} p_{t}^{\mathrm{c}, \min } ; \forall t \\
& E_{t}^{\max }=E_{t-1}^{\max }-p_{t}^{d, \min } / \eta^{d}+\eta^{c} p_{t}^{\mathrm{c}, \max } ; \forall t \\
& \underline{E} \leq E_{t}^{\min } \leq E_{t}^{\max } \leq \bar{E} ; \forall t \\
& \mu_{t}^{c} \underline{p}^{c} \leq p_{t}^{c, \min } \leq p_{t}^{c, \max } \leq \mu_{t}^{c} \bar{p}^{c} ; \forall t \\
& \mu_{t}^{d} \underline{p}^{d} \leq p_{t}^{d, \text { min }} \leq p_{t}^{d, \text { max }} \leq \mu_{t}^{d} \bar{p}^{d} ; \forall t \\
& \mu_{t}^{d}, \mu_{t}^{c} \in\{0,1\}, \mu_{t}^{d}+\mu_{t}^{c} \leq 1 ; \forall t
\end{aligned}
$$

where $C_{t}$ is the electricity price, $\omega_{s}$ is the weighting factor of the selected scenario and the subscript $S$ $\in\{S 1, S 2\}$ represents the index of scenario, $\tilde{p}_{t, s}^{r \Sigma}$ is the actual total output of renewable plants, $p_{t, s}^{c}$ and $p_{t, s}^{d}$ are the charging and discharging power of shared energy storage. Binary variables $\mu_{t}^{c}$ and $\mu_{t}^{d}$ denote the 
charging and discharging status of share energy storage. The auxiliary variables $p_{t}^{c, \min }$ and $p_{t}^{c, \max }$ are the allowable lower and upper bounds of charging power, $p_{t}^{d, \min }$ and $p_{t}^{d, \max }$ denote the permitted discharging level, $E_{t}^{\min }$ and $E_{t}^{\max }$ give the safe ranges of SoC. The parameter $p^{L}$ is the transmission line capacity and $\alpha^{L}$ is the minimal load rate,$\eta^{c}$ and $\eta^{d}$ are the charging and discharging efficiencies, $\underline{E}$ and $\bar{E}$ are the physical lower and upper bounds of SoC, $\underline{p}^{c}$ and $\bar{p}^{c}, \underline{p}^{d}$ and $\bar{p}^{d}$ give the physical ranges of charging and discharging power, respectively. The objective (6a) is to maximize the weighted sum of generating income. Constraint (6b) gives the transmission line capacity constraint and the minimal load rate restrictions. Constraint (6c)-(6d) describe the real renewable output under each selected scenario considering the curtailment level. Expressions (6e)-(6k) are non-anticipative constraints of shared energy storage that enforce the all-scenario feasible of the charging and discharging strategies. Constraint (61) forbids simultaneous charging and discharging. The minimal value expression in (6c) can be transmitted to an equivalent mixed-linear formulation as follows.

$$
\begin{aligned}
& \tilde{p}_{t, S 1}^{r \Sigma}=\bar{p}_{t}^{r a \Sigma}-h_{t} ; \forall t \\
& \tilde{p}_{t, S 2}^{r \Sigma} \leq \bar{p}_{t}^{r a \Sigma}-h_{t} ; \forall t \\
& \tilde{p}_{t, S 2}^{r \Sigma} \leq \underline{p}_{t}^{r a \Sigma} ; \forall t \\
& \tilde{p}_{t, S 2}^{r \Sigma} \geq \bar{p}_{t}^{r a \Sigma}-h_{t}-\bar{p}_{t}^{r a \Sigma}\left(1-z_{t}^{1}\right) ; \forall t \\
& \tilde{p}_{t, S 2}^{r \Sigma} \geq \underline{p}_{t}^{r a \Sigma}-\underline{p}_{t}^{r a \Sigma}\left(1-z_{t}^{2}\right) ; \forall t \\
& z_{t}^{1}, z_{t}^{2} \in\{0,1\}, z_{t}^{1}+z_{t}^{2}=1 ; \forall t
\end{aligned}
$$

where $z_{t}^{1}$ and $z_{t}^{2}$ are induced binary variables.

\subsection{Online dispatch}

In the real-time dispatch stage, after observing the real accessible total output of renewables $p_{t}^{r \Sigma}$, the scheduling of shared energy storage is determined based on the following model.

$$
\begin{array}{cl}
\max _{\tilde{p}_{t}^{r}, p_{t}^{c}, p_{t}^{d}, h_{t}} C_{t}\left(\tilde{p}_{t}^{r \Sigma}-p_{t}^{c}+p_{t}^{d}\right) \\
\text { s.t. } \alpha p^{L} \leq \tilde{p}_{t}^{r \Sigma}-p_{t}^{c}+p_{t}^{d} \leq p^{L} \\
\tilde{p}_{t}^{r \Sigma}=\min \left\{\bar{p}_{t}^{r a \Sigma}-h_{t}, p_{t}^{r \Sigma}\right\} \\
0 \leq h_{t} \leq \bar{p}_{t}^{r a \Sigma} \\
\\
p_{t}^{c, \min *} \leq p_{t}^{c} \leq p_{t}^{c, \text { max* }} \\
p_{t}^{d, \min *} \leq p_{t}^{d} \leq p_{t}^{d, \max *}
\end{array}
$$

where $p_{t}^{c, \text { min* }}$ and $p_{t}^{c, \text { max* }}$ represent the permitted charging ranges determined in the day-ahead stage, $p_{t}^{d, \text { min* }}$ and $p_{t}^{d, \max *}$ are the pre-specified allowable discharging range, and they are the optimal solution to problem (6). It can be proved that if the non-anticipative day-ahead scheduling problem (6) has feasible solutions, then there must be at least one feasible scheduling strategy for the real-time dispatch under all the uncertainty realizations that reside in the pre-specified sets (1) [10-11] Different from the models of multi-period dispatch with energy storage, the time-couping $\mathrm{SoC}$ constraints are relaxed, but the bounds of $\mathrm{SoC}$ will not be violated under any uncertainty realizations due to the pre-defined bounds of charging/discharging power and SoC level. Moreover, the real-time decision-making only depends on the current observations and requires no predictions, which meets online dispatch requirements.

\section{Case study}

Case studies are carried out on an integrated system with 2 wind plants whose capacities are both $400 \mathrm{MW}$, a 400 MW solar plant, and a shared energy storage with the capacity of $200 \mathrm{MW} / 600 \mathrm{MWh}$. Other system parameters are listed in Table. 1. and the predicted renewable output is plotted in Fig.3. All the tests are implemented via Matlab 2016a and Gurobi 9.1.1.

Table1. System parameters.

\begin{tabular}{|c|c|c|c|}
\hline Parameters & Value (unit) & Parameters & $\begin{array}{c}\text { Value } \\
\text { (unit) }\end{array}$ \\
\hline$\alpha^{L}$ & 0.5 & $p^{L}$ & $600 \mathrm{MW}$ \\
\hline$\eta^{c}$ & 0.95 & $\eta^{d}$ & 0.95 \\
\hline$\underline{E}$ & $30 \mathrm{MWh}$ & $\bar{E}$ & $600 \mathrm{MWh}$ \\
\hline$p^{c}$ & $50 \mathrm{MW}$ & $\bar{p}^{c}$ & $200 \mathrm{MW}$ \\
\hline$p^{d}$ & $50 \mathrm{MW}$ & $\bar{p}^{d}$ & $200 \mathrm{MW}$ \\
\hline$C_{t}$ & $\begin{array}{c}0.8 \\
\text { yuan/MWh }\end{array}$ & $E_{0}$ & $180 \mathrm{MWh}$ \\
\hline
\end{tabular}

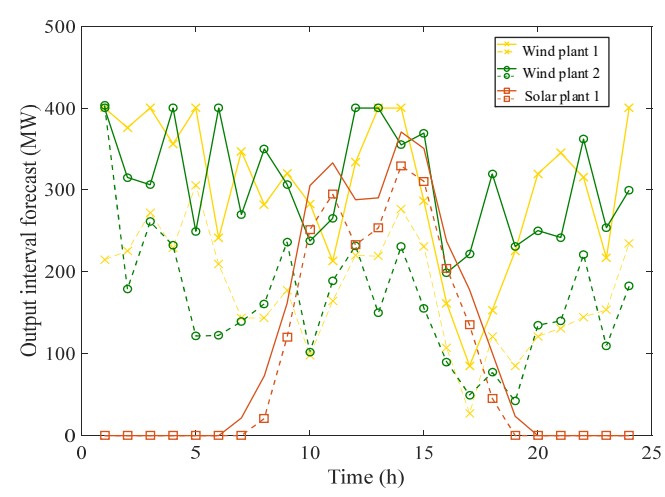

Fig.3 Prediction of renewable output intervals.

The day-ahead scheduling results are shown in Fig. 45. Fig. 4 displays the safe ranges of charging and discharging power; Fig. 5 displays the permitted variations of $\mathrm{SoC}$, which are kept within the physical operation ranges of energy storage, indicating that the adjustment of energy storage can guarantee the minimal load rate constraints and transmission capacity limitation under all the uncertainty realizations. 


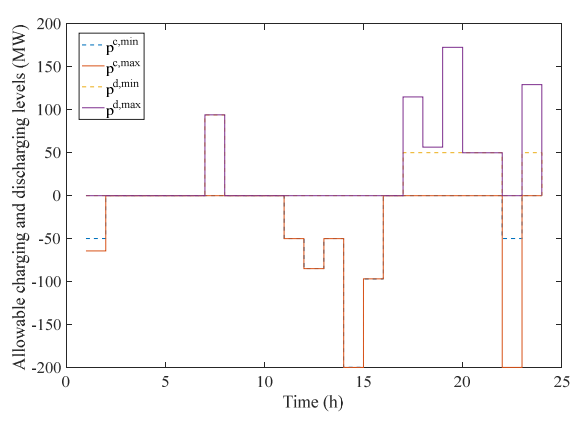

Fig.4 Allowable charging and discharging ranges.

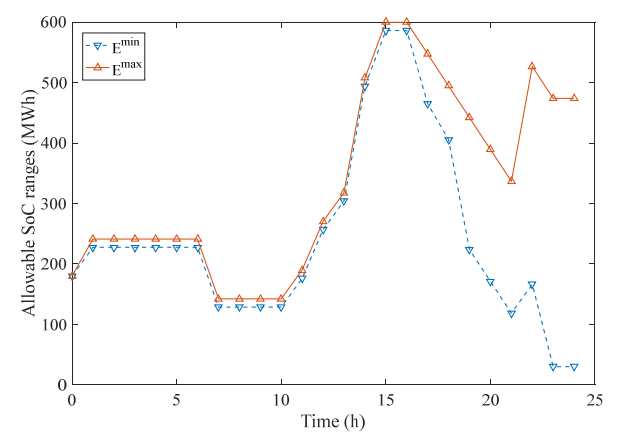

Fig.5 Allowable SoC ranges.

The observed renewable output and the power delivery through the transmission line in the real-time stage are shown in Fig. 6. In periods 11-15, the accessible renewable output exceeds the transmission line capacity, thus part of the surplus is used to charge the shared energy storage and the residual energy is abandoned which is represented by the shadow area in Fig. 6, leading to 257.3 MWh renewable curtailment. In periods $17-21$, the actual total renewable output below the required minimum load, and the vacancy is compensated by the discharging power.

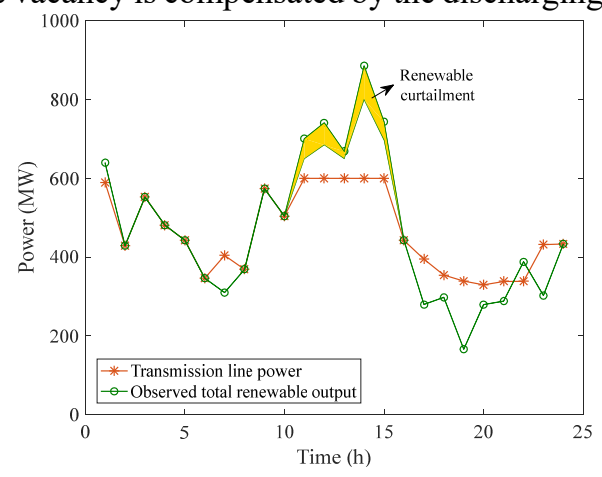

Fig.6 Online dispatch results.

The weighted sum of generating income and total curtailment scheduled in the day-ahead stage with different line capacity settings is plotted in Fig. 7. As can be observed, with the increase of the transmission line capacity, the generation income grows up and the total curtailment decreases. However, considering the minimal load ratio constraints, when transmission line capacity is too large, i.e., more than $630 \mathrm{MW}$, the discharging of energy storage can't fully compensate for the insufficiency of renewables output, leading to infeasible day-ahead solutions.

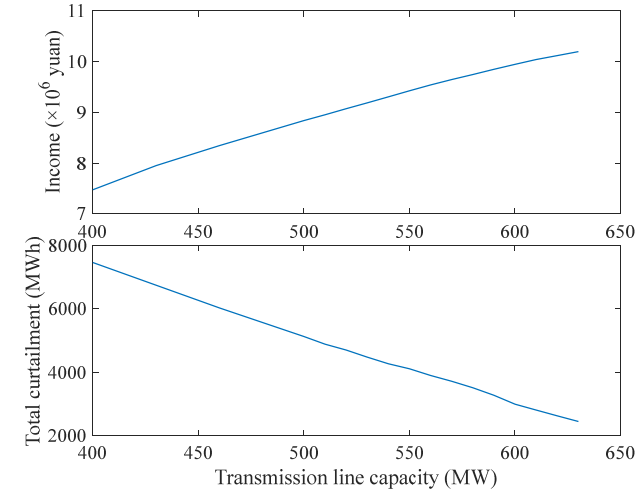

Fig.7 Influence of transmission line capacity.

The weighted sum of generating income and total curtailment scheduled in the day-ahead stage with different minimal load ratios is plotted in Fig. 8. With the increase of minimal load ratio, the generating income decreases because the more renewable output is consumed to charge the energy storage to support the discharging during the periods with inadequate renewable outputs and less renewable energy can be sold; when the minimal load ratio reaches 0.53 , the vacancy cannot be fully compensated by energy storage due to its maximal charging power. When the minimal load ratio is set below 0.45 , the generation income cannot be elevated by the decrease of load ratio; when less than 0.42 , it has little impact on the total curtailment.

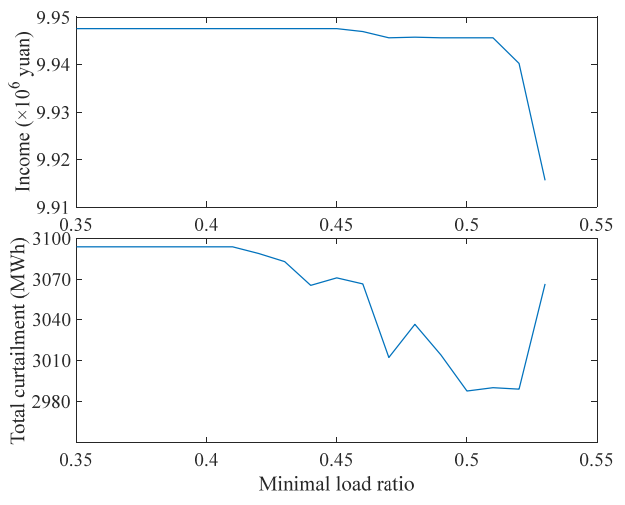

Fig.8 Influence of minimal load ratio.

\section{Conclusion}

This paper explores the online dispatch of a renewablestorage integrated system where a central energy storage is shared by multiple renewable plants. The proposed method guarantees that the total power exported via the transmission line satisfies the requirement on the utilization rate of transmission capacity, regardless of the hourly unfolding real renewable output. Case study shows that the SoC of the shared energy storage is constrained by two additional bounds, implying that the SoC can vary within a subrange of its physical ranges. 


\section{Acknowledgment}

This paper is supported by State Grid Anhui Electric Power Co., Ltd. Science and Technology Project (52120520004M).

\section{References}

1. J. Jo, J. Park, 11, IEEE T. Smart Grid 11, 4466-4476 (2020)

2. R. Dai, R. Esmaeilbeigi, H. Charkhgard, IEEE T. Smart Grid (2021)

3. D. Kim, H. Kim, D. Won, IEEE Access 8, 197097197110 (2020)

4. W. Zhong, K. Xie, Y. Liu, C. Yang, S. Xie, IEEE T. Smart Grid 11, 4105-4115 (2020)

5. P. Huang, Y. Sun, M. Lovati, X. Zhang, Energy 222, 119931 (2021)

6. R. Dai, H. Charkhgard, F. Rigterink, Int. T. Oper. Res. (2020)

7. W. Zhang, W. Wei, L. Chen, B. Zheng, S. Mei, Energy 202, 117543 (2020)

8. M.A. Mirzaei, A.S. Yazdankhah, B. MohammadiIvatloo, M. Marzband, M. Shafie-khah, J.P. Catalão, J. Clean Prod. 223, 747-758 (2019)

9. B. Dey, B. Bhattacharyya, S. Sharma, International Journal of Energy Optimization and Engineering 8, 67-87 (2019)

10. Y. Zhou, Q. Zhai, M. Zhou, X. Li, IEEE T. Sustain. Energy 11, 758-770 (2019)

11. Y. Zhou, Q. Zhai, L. Wu, IEEE T. Sustain. Energy (2020) 